\title{
Tabaquismo durante el embarazo: Efectos en la salud respiratoria infantil*
}

\author{
VIVIANA AGUIRRE C.**
}

\section{Smoking during pregnancy: Effects on respiratory children's health}

The prevalence of smoking among young women of developing countries is 20-30\%, mean while its national prevalence is around 40-55\%. Maternal smoking during pregnancy has been associated with adverse outcomes such as: spontaneous abortions, stillbirth, preterm birth, fetal growth restriction, neurodevelopmental disorders, cancers, sudden infant death syndrome, placental complications and oral-facial cleft. About of respiratory health, children of women who smoke during pregnancy are at increased risk of hospitalization, lower respiratory illness, wheezing during childhood, physician-diagnosed asthma, bronchial hyperresponsiveness and chronic deficits in lung function had a more pronounced effect on forced expiratory flows. Reduction of smoking-related adverse pregnancy outcomes requires that existing primary preventive tools, aimed to prevent youth smoking and cessation of smoking among women of childbearing age.

Key words: Tobacco, pregnancy, respiratory health.

\section{Resumen}

La prevalencia de tabaquismo en mujeres en edad fértil alcanza cifras entre 20-30\% en países desarrollados, con prevalencias nacionales entre 40-55\%. Fumar cigarrillo durante el embarazo ha sido implicado en diversas patologías obstétricas y neonatales, tales como desprendimiento de placenta, placenta previa, embarazo ectópico, aborto espontáneo, parto prematuro, mortinato, síndrome de distrés respiratorio del neonato, bajo peso de nacimiento, síndrome de muerte súbita, alteraciones neurocognitivas y defectos palatinos. Respecto a su efecto en la salud respiratoria, el hábito tabáquico durante el embarazo provoca alteración de la función de la vía aérea expresada por flujos espiratorios disminuidos, mayor prevalencia de sibilancias recurrentes y diagnóstico formal de asma, hiperreactividad bronquial, mayor frecuencia de hospitalización e infección respiratoria baja. Enfocándolo como un problema de salud pública, el gran desafío es diseñar y aplicar estrategias efectivas tendientes a evitar la exposición del feto y lactante a los productos derivados del cigarrillo.

Palabras clave: tabaco, embarazo, salud respiratoria.

El tabaquismo constituye un problema mundial de salud por su alta morbi-mortalidad asociada y desafortunadamente también afecta a un porcentaje significativamente alto de mujeres embarazadas. Hay creciente evidencia que indica que este hábito durante el embarazo es res- ponsable de un gran número de patologías obstétricas, neonatales y del desarrollo. La exposición in útero al cigarrillo debe ser considerada como una forma diferente de exposición pasiva al cigarrillo, puesto que el feto no está directamente expuesto al humo de cigarrillo. Las con-

* $\quad$ Basado en la Conferencia: "Tabaquismo durante el embarazo: efectos en la salud respiratoria infantil", pronunciada en las XV Jornadas de Invierno "Contaminación, tabaco y salud" de la Sociedad Chilena de Enfermedades Respiratorias, el 10 de junio de 2006 en Santiago.

** Neumóloga Pediatra, Departamento de Medicina Respiratoria Infantil Hospital CRS El Pino. Universidad de Santiago de Chile (USACH) y Universidad Nacional Andrés Bello (UNAB). 
centraciones de nicotina y cotinina en mujeres fumadoras durante el embarazo y en sus hijos al nacer, indican que los hijos de fumadoras tienen exposición sistémica a las toxinas del tabaco, y que el feto está expuesto desde el momento de la concepción a los mismos niveles de nicotina que el fumador activo. Aunque la nicotina ha sido el foco principal de estudios en animales en este tópico, el humo de cigarrillo está compuesto de cientos de químicos y la contribución individual de cada uno de ellos es desconocida.

El objetivo de este artículo es revisar la evidencia que existe sobre el efecto del tabaquismo en el embarazo, especialmente en el aparato respiratorio del producto de la gestación.

\section{Estadísticas generales de consumo de tabaco en el embarazo y en mujeres en edad fértil}

La prevalencia de tabaquismo en las mujeres jóvenes varía entre los distintos países. Los datos provenientes desde países desarrollados, como EE.UU., Suecia, Dinamarca y el Reino Unido describen una tendencia al descenso en los últimos años, con cifras actuales que oscilan entre el 20-30\% ${ }^{1,2}$; sin embargo, la edad de inicio del hábito de fumar es cada vez menor ${ }^{3}$. En estos países, se ha observado que la prevalencia en las mujeres embarazadas varía según la edad, siendo las mujeres mayores de 30 años las que menos fuman, por el contrario, las más fumadoras se encuentran en el rango de 15 a 24 años ${ }^{1,2}$. Algunos estudios europeos informan que entre el 20-40\% de las fumadoras abandonan el cigarrillo durante el embarazo ${ }^{4-6}$ y que los factores de riesgo más importantes para persistir en el hábito son la multiparidad, la baja educación, el inicio del hábito precozmente en la vida, la condición de ser grandes fumadoras y la presencia de fumadores en la casa o en el trabajo, especialmente si la pareja también fuma ${ }^{4-7}$.

Los datos entregados por el Consejo Nacional para el Control de Estupefacientes (CONACE), indican que en Chile las mujeres entre 19-25 años han aumentado el consumo de cigarrillos en 12 puntos porcentuales entre 1994-2004, alcanzando prevalencia por mes de $55 \%$ en el año 2004. Entre las adultas de 26-34 años, el consumo de cigarrillos se ha mantenido estable en alrededor de un $45 \%$, no obstante se observan leves aumentos en el consumo de cigarrillos en el bienio 2002-2004 ${ }^{8}$. Un reciente estudio chileno de prevalencia de tabaquismo en el embarazo informa de una cifra de $28 \%$.

\section{Efectos generales en el producto de la gestación}

Los efectos del tabaco durante el embarazo (TE) son varios, siendo los más importantes el desprendimiento de placenta, placenta previa, embarazo ectópico, aborto espontáneo, parto prematuro y mortinato ${ }^{3}$. Aunque se describe un efecto protector del TE en el riesgo de desarrollar hipertensión gestacional, cuando la fumadora desarrolla pre-eclampsia, la hipoxia placentaria puede ser extremadamente pronunciada, llevando a riesgos sustancialmente aumentados de hipoxia fetal, desprendimiento de placenta y muerte fetal $^{10}$.

Se estima que el TE sería el responsable de cerca del $18 \%$ de los casos de bajo peso de nacimiento $(<2.500 \mathrm{~g})$, de un riesgo aumentado de muerte súbita del lactante, y un mayor riesgo de mortalidad perinatal ${ }^{1,3,11}$. Además, se le ha asociado con un mayor riesgo de alteraciones neurocognitivas ${ }^{12}$, cánceres $^{13}$ y malformaciones congénitas en la niñez ${ }^{14}$.

\section{Mecanismos de acción involucrados}

Aunque los mecanismos por los cuales el tabaco provoca daño en el feto no están del todo claros, existen trabajos que correlacionan alteraciones específicas con ciertas patologías. El cigarrillo provoca transitoriamente descensos en el flujo de sangre uterina materna, reduciendo de esta forma, el flujo de oxígeno desde el útero a la placenta. Los elevados niveles de carboxihemoglobina que se encuentran tanto en la sangre materna y fetal cuando la madre fuma en el embarazo, pueden llevar a hipoxia fetal crónica evidenciada por hematocrito aumentado en el recién nacido ${ }^{15-17}$. El retardo en el crecimiento intrauterino puede ser atribuido a varios factores incluyendo las propiedades vasoconstrictoras de la nicotina, elevación de los niveles de carboxihemoglobina y de catecolaminas, hipoxia tisular fetal, reducción de la liberación de elementos nutricionales fetales, elevación de la frecuencia cardíaca y de la presión sanguínea ${ }^{18}$. En el caso de lactantes con muerte súbita se ha observado un aumento en el grosor de la vía aérea asociado con el tabaco materno de más de 20 cigarrillos diarios ${ }^{19}$. Elliot y $\operatorname{cols}^{20}$, han descrito una mayor distancia entre los puntos de unión alveolar en la vía aérea intraparenquimatosa en lactantes expuestos a TE comparados con los no expuestos y con los lactantes expuestos solamente en el período postnatal, sugiriendo que 
esto podría ser el resultado de un desarrollo disminuido de los alvéolos in útero y durante el período postnatal, expresándose en una reducción en la retracción elástica pulmonar. Lo anterior concuerda con el hecho que el riesgo de muerte súbita es mayor en lactantes expuestos a tabaco en el embarazo y en período postnatal que en los expuestos solamente en el período postnatal ${ }^{21}$.

Con respecto a la prevalencia aumentada de síntomas respiratorios en hijos de madres fumadoras, estudios en animales y en humanos han demostrado que la exposición del feto a la nicotina se asocia con varias anormalidades morfológicas significativas del pulmón: bronquios tortuosos y dilatados, conformación anormal de la superficie de la mucosa de los bronquios pequeños, aumento en el tamaño y composición celular de los cuerpos neuroepiteliales, disminución en el peso del pulmón fetal, volumen pulmonar fetal disminuido, disminución en el número y tamaño de los alvéolos fetales y disminución del volumen total del tejido elástico parenquimatoso ${ }^{22-25}$. Esta información sugiere que la exposición del feto al TE afecta predominantemente el desarrollo de la vía aérea intratorácica, sin embargo, el efecto general es una alteración intrauterina del crecimiento y maduración pulmonar. Aunque el TE ha demostrado tener un efecto en el crecimiento somático, la reducción en el flujo aéreo no se explicaría totalmente por la reducción en el crecimiento somático ${ }^{26}$.

El tabaquismo materno también alteraría la función endocrina relacionada con la maduración del sistema respiratorio ${ }^{27-29}$, habiéndose demostrado una maduración pulmonar acelerada, expresada por un mayor nivel de cortisol en el líquido amniótico, en fetos expuestos al cigarri$1 \mathrm{lo}^{27}$.

Con respecto al asma y TE, los mecanismos biológicos involucrados no están debidamente aclarados. Es posible que el daño precoz inducido por el tabaquismo materno en la pequeña vía aérea (PVA) del feto, en un período crítico de su desarrollo, altere permanentemente tanto la función de la PVA como la retracción elástica y la función inmune ${ }^{30}$. En roedores neonatos expuestos al humo de tabaco en el período neonatal se ha encontrado un aumento en la reactividad bronquial ${ }^{31}$, lo que podría conducir tanto a un aumento en el riesgo de asma como a alteraciones en los flujos de la vía aérea. El efecto también puede ser secundario al aumento de problemas respiratorios neonatales o infecciones precoces asociadas al $\mathrm{TE}^{32}$.

\section{Efectos en la salud respiratoria y la función pulmonar}

No ha sido fácil diferenciar si el efecto adverso del cigarrillo en la salud del niño es atribuible a un daño en el desarrollo del feto in útero o a la exposición al humo de tabaco después del nacimiento. Lo anterior es especialmente debido a que la mayoría de las mujeres que fuman en el embarazo, continúan fumando después del parto y también por la alta prevalencia de exposición pasiva al humo de tabaco en el período postnatal ${ }^{3}$. Los estudios en neonatos y lactantes, cuyas madres fumaron en el embarazo, demuestran una reducción importante de los flujos espiratorios, proporcionando evidencia indirecta de la disminución del calibre de la vía aérea y ocasionalmente reducción leve de la capacidad residual funcional (FRC) ${ }^{26,33-38}$. Los trabajos que han logrado separar la exposición in útero de aquella postnatal, ya sea porque han evaluado precozmente al paciente o porque las madres han dejado de fumar después del parto, han informado de una mayor prevalencia de sibilancias recurrentes durante los 2-3 primeros años de vida, principalmente si la exposición fue durante las etapas más precoces del embarazo $^{39,40}$ y especialmente en individuos genéticamente susceptibles ${ }^{41}$. Milner y cols ${ }^{42}$, evaluó precozmente a neonatos expuestos in útero, antes de ser expuestos al cigarrillo ambiental y demostró que el cigarrillo en el embarazo tenía un efecto adverso en el peso de nacimiento, talla, circunferencia de cráneo y tórax; con una menor distensibilidad de los pulmones de los neonatos masculinos y una mayor resistencia de la vía aérea en los pulmones de los neonatos femeninos.

En nuestro hospital, evaluamos 148 lactantes portadores de sibilancias recurrentes, mediante espiración forzada con volumen pulmonar aumentado (raised volume) ${ }^{43}$ y pudimos observar una disminución significativa de los flujos en el $50 \%\left(\mathrm{FEF}_{50}\right)$ y $25-75 \%\left(\mathrm{FEF}_{25-75}\right)$ de la capacidad vital forzada en los lactantes cuyas madres tenían el antecedente de tabaquismo en el embarazo. Al evaluar la presencia de tabaco intradomiciliario en la función pulmonar de estos lactantes, no encontramos diferencias entre los expuestos y no expuestos, apoyando lo encontrado en otros trabajos que sugieren que en los primeros años de vida, la exposición al tabaco en el embarazo tiene una mayor importancia que la exposición pasiva postnatal al cigarrillo ${ }^{44}$.

Durante los primeros años de vida, los niños de madres que fumaron en el embarazo tienen 
un mayor riesgo de hospitalización ${ }^{45-49}$ y mayor riesgo de infección respiratoria baja ${ }^{45,47,49,50}$, sugiriendo que el efecto del tabaco podría alterar la respuesta inmune frente a las infecciones por lo menos durante el primer año de vida, como se infiere de trabajos recientes que demuestran que el TE provocaría una modificación de la función de células fetales Th1 y Th2 e inhibición de la inmunidad innata a una variedad de "toll-like receptors" (TLR) $)^{51,52}$.

Hay evidencia que sugiere que el efecto deletéreo del TE puede alcanzar hasta la edad escolar; un meta-análisis de 21 estudios encontró una reducción del $\mathrm{VEF}_{1}$ de 1,4\%, del $\mathrm{FEF}_{25-75}$ de $5 \%$ y tasas de flujo al final de la espiración reducidas en $4,3 \%$ en escolares expuestos pasivamente al cigarrillo, no diferenciando la exposición prenatal de la postnatal ${ }^{30}$. En escolares y adolescentes cuyas madres fumaron en el embarazo, pero no lo hicieron después del parto se ha podido demostrar una disminución significativa de los flujos espiratorios en comparación con los hijos de madres que nunca fumaron pero cuyos niños estuvieron expuestos al tabaco ambiental después del parto ${ }^{33}$. También se ha encontrado que tanto los valores espirométricos como la capacidad de difusión pulmonar (DLco) son más bajos en niños expuestos in útero y después del nacimiento, comparado con los que estuvieron expuestos al tabaco ambiental solamente después del parto; en este último subgrupo los valores mencionados fueron más bajos que en aquellos niños sin ningún tipo de exposición al tabaco, sugiriendo que ambas exposiciones son factores independientes en la determinación del daño pulmonar ${ }^{53}$. Por otra parte, se ha observado que los efectos en la función pulmonar de la exposición al TE son mayores en niños que tienen una historia familiar de asma ${ }^{35}$, y que estos déficit en la función pulmonar persisten hasta la adolescencia ${ }^{32-35,37,53-57}$, especialmente en los parámetros de flujo aéreo.

Gilliland y cols ${ }^{59}$, demostraron que el TE aumenta la ocurrencia de asma diagnosticada por médico y sibilancias durante la niñez, a diferencia de la exposición pasiva al cigarrillo después del nacimiento, la cual se asoció sólo con sibilancias, pero no con diagnóstico formal de asma. Más aún, entre los niños que estuvieron expuestos tanto in útero como postnatal, la exposición postnatal no aumentó la incidencia de asma o sibilancias más allá que la asociada con la exposición in útero. Tomados en conjunto, los trabajos en esta área sugieren que la exposición pasiva al tabaco ambiental después del parto actuaría como cofactor con infecciones intercurren- tes para desencadenar ataques de sibilancias, mientras que la exposición in útero actúa aumentando el diagnóstico formal de asma.

Las alteraciones persistentes en la función de la vía aérea pequeña derivadas de una exposición al tabaco in útero pueden predisponer a los niños a sibilar durante las infecciones respiratorias $\mathrm{u}$ otros insultos que producen inflamación, subsecuente hiperreactividad bronquial (HRB) y obstrucción al flujo aéreo. Además, los estudios en neonatos humanos demuestran que la exposición in útero al tabaco se asocia con HRB aumentada, especialmente en aquellos con una historia familiar de asma ${ }^{60}$. Una reactividad bronquial crónicamente aumentada desde la exposición in útero sumado a otras noxas postnatales puede ser un factor de riesgo para sibilancias persistentes y para una predisposición aumentada al asma. De acuerdo a la información disponible, se podría inferir que la eliminación del TE podría prevenir $5-15 \%$ de los casos de asma en niños ${ }^{59}$.

El desarrollo fetal representa un tiempo crítico de vulnerabilidad somática y funcional siendo de gran importancia proteger al producto de daños evitables durante este período. Es sorprendente que las diferentes estrategias empleadas para evitar el consumo de tabaco en el embarazo no hayan tenido un gran impacto en la cesación del hábito, independientemente del nivel socioeconómico o cultural de las madres. Lo anterior hace necesario un enfoque diferente del problema que permita identificar y emplear nuevas herramientas destinadas a desincentivar el consumo de tabaco por mujeres en edad fértil, embarazadas y sus familiares al interior de las viviendas o locales de trabajo. El gran desafío, a la luz de la evidencia actual, es implementar programas gubernamentales y privados destinados a evitar en forma eficiente la exposición del feto y del lactante a los productos derivados del cigarrillo (ej. educación a las niñas en edades precoces de la vida).

\section{Conclusiones}

De lo expuesto anteriormente podemos concluir que el tabaquismo materno durante el embarazo representa una injuria importante tanto para el desarrollo del proceso de gestación como para el producto de ésta. Es en el período neonatal y durante los primeros años de vida donde se manifiestan los mayores efectos respiratorios derivados de la exposición in útero al tabaco, con una mayor incidencia de síndrome de difi- 
cultad respiratoria neonatal, de muerte súbita, sibilancias recurrentes, hiperreactividad bronquial, aumento del riesgo de hospitalización, infecciones respiratorias bajas y mayor incidencia de asma en la niñez y adolescencia.

\section{Bibliografía}

1.- CNATTINGIUS S. The epidemiology of smoking during pregnancy: smoking prevalence, maternal characteristics, and pregnancy outcomes. Nicotine Tob Res 2004; 6: S125-S140.

2.- OWEN L, MCNEILL A, CALLUM C. Trends in smoking during pregnancy in England, 1992-7: quota sampling surveys. BMJ 1998; 317: 728-30.

3.- The health consequences of tobacco use among women. In women and smoking: a report of the surgeon general. Rockville Md: US Dept of Health and Human Services 2001; 177-450.

4.- CNATTINGIUS S, LINDMARK G, MEIRIK O. Who continues to smoke while pregnant? J Epidemiol Community Health 1992; 46: 218-21.

5.- FINGERHUT L A, KLEINMAN J, KENDRICK J S. Smoking before, during and after pregnancy. Am J Public Health 1990; 80: 541-4.

6.- WISBORG K, HENRIKSEN T B, HEDEGAARD M, SECHER N J. Smoking among pregnant women and the significance of socio-demographic factors on smoking cessation. Ugeskr Laeger 1996; 158: 3784-8.

7.- TORRENT M, SUNYER J, CULLINAN P, BASAGAÑA X, HARRIS J, GARCÍA O, et al. Smoking cessation and associated factors during pregnancy. Gac Sanit 2004; 18: 184-9.

8.- www.conacedrogas.cl/inicio/pdf/Consumo_cigarrillos_ Chile_CONACEmayo2005.pdf

9.- MALLOL J, BRANDENBURG D, MADRID R, SEMPERTEGUI F, RAMÍREZ L, JORQUERA D. Prevalencia de tabaquismo durante el embarazo en mujeres chilenas de bajo nivel socioeconómico. Rev Chil Enf Respir 2007; 23: 17-22.

10.- CNATTINGIUS S, MILLS J L, YUEN J, ERIKSSON O, SALONEN H. The paradoxical effect of smoking in preeclamptic pregnancies: Smoking reduces the incidence but increases the rates of perinatal mortality, abruptio placentae, and intrauterine growth restriction. Am J Obstet Gynecol 1997; 177: 156-61.

11.- DIFRANZA J R, ALIGNE C A, WEITZMAN M. Prenatal and postnatal environmental tobacco smoke exposure and children's health. Pediatrics 2004; 113: 1007-15.

12.- FERGUSSON D M, WOODWARD L J, HORWOOD L J. Maternal smoking during pregnancy and psychiatric adjustment in late adolescence. Arch Gen Psychiatry 1998; 55: 721-7.

13.- SCHWARTZBAUM J A, GEORGE S L, PRATT C B, DAVIS B. An exploratory study of environmental and medical factors potentially related to childhood cancer. Med Pediatr Oncol 1991; 19: 115-21.

14.- WYSZYNSKI D F, DUFFY D L, BEATY T H. Maternal cigarette smoking and oral clefts: a meta-analysis. Cleft Palate Craniofac J 1997; 34: 206-10.

15.- ULM M R, PLOCKINGER B, PIRICH C, GRYGLEWSKI R J, SINZINGER H F. Umbilical arteries of babies born to cigarette smokers generate less prostacyclin and contain less arginine and citrulline compared with those of babies born to control subjects. Am J Obstet Gynecol 1995; 172: 1485-7.

16.- JAUNIAUX E, BIERNAUX V, GERLO E, GULBIS B. Chronic maternal smoking and cord blood amino acid and enzyme levels at term. Obstet Gynecol 2001; 97: 57-61.

17.- BUSH P G, MAYHEW T M, ABRAMOVICH D R, AGGETT P J, BURKE M D, PAGE K R. Maternal cigarette smoking and oxygen diffusion across the placenta. Placenta 2000; 21: 824-33.

18.- MONER S. Smoking and Pregnancy. In: Canadian Task Force on the Periodic Health Examination. Canadian Guide to Clinical Preventive Health Care. Ottawa: Health Canada, 1994; 26-36.

19.- ELLIOT J, VULLERMIN P, ROBINSON P. Maternal cigarette smoking is associated with increased inner airway wall thickness in children who die from sudden infant death syndrome. Am J Respir Crit Care Med 1998; 158: 802-6.

20.- ELLIOT J G, CARROLL N G, JAMES A L, ROBINSON P J. Airway alveolar attachment points and exposure to cigarette smoke in utero. Am J Respir Crit Care Med 2003; 167: 45-9.

21.- GIDDING S S, SCHYDLOWER M. Active and passive tobacco exposure: a serious pediatric health problem. Pediatrics 1994; 94: 750-1.

22.- BASSI J A, ROSSO P, MOESSINGER A C, BLANC W A, JAMES L S. Fetal growth retardation due to maternal tobacco smoke exposure in the rat. Pediatr Res 1984; 18: 127-30.

23.- CHEN M F, KIMIZUKA G, WANG N S. Human fetal lung changes associated with maternal smoking during pregnancy. Pediatr Pulmonol 1987; 3: 51-8.

24.- COLLINS M, MOESSINGER A C, KLEINERMAN J, BASSI J, ROSSO P, COLLINS A M, et al. Fetal lung hypoplasia associated with maternal smoking: a morphometric analysis. Pediatr Res 1985; 19: 408-12.

25.- WANG N, SCHARAUFNAGEL D, CHEN M. The effect of maternal oral intake of nicotine on the growth and maturation of fetal and baby mouse lungs. Lung 1983; 161: 27-38.

26.- YOUNG S, SHERRILL D L, ARNOTT J, DIEPEVEEN D, LESOUËF P N, LANDAU L I. Parental factors affecting respiratory function during the first year of life. Pediatr Pulmonol 2000; 29: 331-40.

27.- LIEBERMAN E, TORDAY J, BARBIERI R, COHEN A, VAN VUNAKIS H, WEISS S T. Association of intrauterine cigarette smoke exposure with indices of fetal lung maturation. Obstet Gynecol 1992; 79: 564-70.

28.- CATLIN E A, POWELL S M, MANGANARO T F, HUDSON P L, RAGIN R C, EPSTEIN J, et al. Sexspecific fetal lung development and mullerian inhibiting substance. Am Rev Respir Dis 1990; 141: 466-70.

29.- DIVERS W A, WILKES M M, BABAKNIA A, YEN S S. Maternal smoking and elevation of catecholamines and metabolites in the amniotic fluid. Am J Obstet Gynecol 1981; 141: 625-8.

30.- COOK D G, STRACHAN D P, CAREY I M. Health effects of passive smoking. 9. Parental smoking and spirometric indices in children. Thorax 1998; 53: 88493.

31.- JOAD J P, BRIC J M, PEAKE J L, PINKERTON K E. Perinatal exposure to aged and diluted sidestream cigarette smoke produce airway hyperresponsiveness in older rats. Toxicol Appl Pharmacol 1999; 155: 253-60.

32.- SHERRILL D L, MARTÍNEZ F D, LEBOWITZ M D, HOLDAWAY M D, FLANNERY E M, HERBISON G 
$\mathrm{P}$, et al. Longitudinal effects of passive smoking on pulmonary function in New Zealand children. Am Rev Respir Dis 1992; 145: 1136-41.

33.- TAGER I B, NGO L, HANRAHAN J P. Maternal smoking during pregnancy. Effects on lung function during the first 18 months of life. Am J Respir Crit Care Med 1995; 152: 977-83.

34.- HANRAHAN J P, TAGER I B, SEGAL M R, TOSTESON T D, CASTILE R G, VAN VUNAKIS H, et al. The effect of maternal smoking during pregnancy on early infant lung function. Am Rev Respir Dis 1992; 145: 1129-35.

35.- STICK S M, BURTON P R, GURRIN L, SLY P D, LESÖUEF P N. Effects of maternal smoking during pregnancy and a family history of asthma on respiratory function in newborn infants. Lancet 1996; 348: $1060-4$.

36.- LODRUP CARLSEN K C, JAAKKOLA J J, NAFSTAD $\mathrm{P}$, CARLSEN K H. In utero exposure to cigarette smoking influences lung function at birth. Eur Respir J 1997; 10: 1774-9.

37.- HANRAHAN J P, HALONEN M. Antenatal interventions in childhood asthma. Eur Respir J Suppl 1998; 27: 46s-51s

38.- CUNNINGHAM J, DOCKERY D W, SPEIZER F E. Maternal smoking during pregnancy as predictor of lung function in children. Am J Epidemiol 1994; 139: 1139-52.

39.- LANNERÖ E, WICKMAN M, PERSHAGEN G, NORDWALL L. Maternal smoking during pregnancy increases the risk of recurrent wheezing during the first years of life (BAMSE). Respir Res 2006; 7: 3.

40.- LUX A L, HENDERSON A J, POCOCK S J and THE ALSPAC STUDY TEAM. Wheeze associated with prenatal tobacco smoke exposure: a prospective, longitudinal study. Arch Dis Child 2000; 83: 307-12.

41.- GILLILAND F D, LI Y F, DUBEAU L, BERHANE K, AVOL E, MCCONNELL R, et al. Effects of glutathione S-tranferase M1, maternal smoking during pregnancy, and envrironmental tobacco smoke on asthma and wheezing in children. Am J Respir Crit Care Med 2002; 166: 457-63.

42.- MILNER A D, MARSH M J, INGRAM D M, FOX G F, SUSIVA C. Effects of smoking in pregnancy on neonatal lung function. Arch Dis Child Fetal Neonatal Ed 1999; 80: F8-14.

43.- MALLOL J, AGUIRRE V. Advances in the Study of Infant Lung Function: Forced Expiratory Maneuvers From an Increased Lung Volume. Arch Bronconeumol 2007; 43: 233-8.

44.- WANDALSEN G, AGUIRRE V, MALLOL J. Espirometria em lactentes com sibilância recorrente. Rev Bras Alerg Inmunopatol 2003; 26: 41-52.

45.- HARLAP S, DAVIES A M. Infants admission to hospital and maternal smoking. Lancet 1974; 1: 529-32.

46.- RANTAKALLIO P. Relationship of maternal smoking to morbidity and mortality of the child up to the age of five. Acta Paediatr Scand 1978; 67: 621-31.

47.- TAYLOR B, WADSWORTH J. Maternal smoking during pregnancy and lower respiratory tract illness in early life. Arch Dis Child 1987; 62: 786-91.

48.- WEITZMAN M, GORTMAKER S, WALKER D K, SOBOL A. Maternal smoking and childhood asthma. Pediatrics 1990; 85: 505-11.

49.- WISBORG K, HENRIKSEN T B, OBEL C, SKAJAA E, OSTERGAARD J R. Smoking during pregnancy and hospitalization of the child. Pediatrics 1999; 104: e46.

50.- MALLOL J, KOCH E, CARO N, SEMPERTEGUI F, MADRID R. Prevalencia de enfermedades respiratorias en el primer año de vida en hijos de madres que fumaron durante el embarazo. Rev Chil Enf Respir 2007; 23: 23-9.

51.- NOAKES P S, HALE J, THOMAS R, LANE C, DEVADASON S G, PRESCOTT S L. Maternal smoking is associated with impaired neonatal toll-likereceptor mediated immune responses. Eur Respir J 2006; 28: 721-9.

52.- LE SÖUEF P N. Adverse effects of maternal smoking during pregnancy on innate immunity in infants. Eur Respir J 2006; 28: 675-7.

53.- RIZZI M, SERGI M, ANDREOLI A, PECIS M, BRUSCHI C, FANFULLA F. Environmental tobacco smoke may induce early lung damage in healthy male adolescents. Chest 2004; 125: 1387-93.

54.- LI Y, GILLILAND F D, BERHANE K, MCCONNELL $\mathrm{R}$, GAUDERMAN W J, RAPPAPORT E B, et al. Effects of in utero and environmental tobacco smoke exposure on lung function in boys and girls with and without asthma. Am J Respir Crit Care Med 2000; 162: 2097-104.

55.- GILLILAND F D, BERHANE K, LI Y F, RAPPAPORT E B, PETERS J M. Effects of early onset asthma and in utero exposure to maternal smoking on childhood lung function. Am J Respir Crit Care Med 2003; 167: 917-24.

56.- WANG $\mathrm{X}$, WYPIJ D, GOLD D R, SPEIZER F E, WARE J H, FERRIS B G, et al. A longitudinal study of the effects of parental smoking on pulmonary function in children 6-18 years. Am J Respir Crit Care Med 1994; 149: 1420-5.

57.- CUNNINGHAM J, O'CONNOR G T, DOCKERY D W, SPEIZER F E. Environmental tobacco smoke, wheezing and asthma in children in 24 communities. Am J Respir Crit Care Med 1996; 153: 218-24.

58.- TAGER I, SEGAL M R, MUNOZ A, WEISS S T, SPEIZER F E. The effect of maternal cigarette smoking on the pulmonary function of children and adolescents: analyses data from two populations. Am Rev Respir Dis 1987; 136: 1366-70.

59.- GILLILAND F D, LI Y F, PETERS J M. Effects of maternal smoking during pregnancy and environmental tobacco smoke on asthma and wheezing in children. Am J Respir Crit Care Med 2001; 163: 429-36.

60.- YOUNG S, LE SOUËF P N, GEELHOED G C, STICK S M, TURNER K J, LANDAU L I. The influence of a family history of asthma and parental smoking on airway responsiveness in early infancy. $\mathrm{N}$ Engl J Med 1991; 324: 1168-73.
Correspondencia a:

Dra. Viviana Aguirre Camposano

Avda. Padre Hurtado (ex Los Morros) 13560

San Bernardo. Santiago, Chile

Fono: 3874694 - Fax 3874662

E-mail: vaguirrecam@yahoo.com 